\title{
Subcortical arteriosclerotic encephalopathy: a clinical and radiological investigation
}

\author{
L A LOIZOU*, B E KENDALL, A ND J M A R H A L
}

From the Department of Neurology, Queen Elizabeth Hospital, Birmingham, and the Lysholm Department of Neuroradiology and Institute of Neurology, the National Hospital, London

S U M MAR Y Subcortical arteriosclerotic encephalopathy (Binswanger's type) was diagnosed in a group of patients with hypertension or arteriosclerosis, who showed acute and subacute neurological deficits, dementia, reduced cerebral blood flow, and white matter low attenuation with mild atrophy and infarcts as the predominant CT scan features. This set of clinical and radiological criteria could be used to make the diagnosis in life, as confirmed neuropathologically in one patient.

In a comprehensive review article, Olszewski ${ }^{1} \mathrm{de}-$ fined the pathological features of subcortical arteriosclerotic encephalopathy, which he considered to be a special form of cerebral arteriosclerosis. In this condition, arteriosclerotic changes affect predominantly the perforating vessels to the subcortical grey substance and white matter, leading to multiple small areas of infarction with diffuse areas of demyelination in the latter; there is relative sparing of the subcortical arcuate fibres and cortex, and in some cases other arteriosclerotic lesions, such as basal ganglia infarcts may be found. Olszewski based this definition on the observations he made on two cases, and after careful consideration of the available literature. The clinical features of patients shown to have had these neuropathological changes included a slowly developing dementia in the 50-60 year old age group, associated with focal neurological deficits, and psychiatric disturbances in some. Binswanger ${ }^{2}$ was in fact the first to describe a group of eight patients with such a clinical picture under the term "Encephalitis subcorticalis chronica progressiva." He attributed the cause of subcortical white matter degeneration to deficient blood supply resulting from atherosclerosis, but he failed to support his views with histological examination of the brains; he merely described the macroscopic

\footnotetext{
* Present Address: The Midland Centre for Neurosurgery and Neurology, Holly Lane, Smethwick, West Midlands.

Address for correspondence and reprint requests: Professor J Marshall, Institute of Neurology, The National Hospital, Queen Square, London, WC1N 3BG.

Accepted 10 February 1981
}

features of the brain of one patient who also had syphilis.

Until recently the diagnosis of this disorder has been based on neuropathological data, the clinical features being common to other disorders, such as multi-infarct dementia ${ }^{3}$ or even Alzheimer's dementia. Biemond ${ }^{4}$ was the first to propose a scheme, on which the diagnosis could be made in life. A comprehensive account of the clinical features was subsequently given by Caplan and Schoene, ${ }^{5}$ based on their observations of neuropathologically proven cases and clinical assessment of a further six cases. Their patients' clinical features included hypertension, acute strokes with or without some recovery, subacute deficits, long plateau periods, lengthy course, dementia, prominent motor signs and pseudobulbar palsy and hydrocephalus. This was a significant contribution to the understanding of subcortical arteriosclerotic encephalopathy, as previous reports in the English literature had referred to a small number of cases only. ${ }^{1} 4 ;-11$

A further contribution to the premorbid diagnosis of this condition has been provided by the use of computerised tomography (CT). Rosenberg, Kornfeld, Stovring and Bicknell ${ }^{12}$ described the clinical, CT and neuropathological features of a patient with subcortical arteriosclerotic encephalopathy. Zeumer, Schonsky and Sturm ${ }^{13}$ described briefly a similar case and a further five patients in whom white matter low attenuation was the main CT scan correlate of a clinical picture of dementia, focal neurological deficits and hypertension. Loizou and Smith (in preparation) have 
studied a 49-year-old normotensive man, with clinical and neuropathological features of subcortical arteriosclerotic encephalopathy, in whom the CT scan showed a combination of subcortical white matter low attenuation, cortical and subcortical infarcts, and no cortical atrophy.

In the present study we attempted to identify clinically patients with dementia of vascular aetiology, in whom other features suggested subcortical arteriosclerotic encephalopathy; we supported the clinical diagnosis by finding the characteristic CT appearances of white matter low attenuation; we obtained further information by psychometry and cerebral blood flow studies, to supplement the clinical and radiological features, in the anticipation that a precise definition of the syndrome could then be made. Neuropathological confirmation of the diagnosis has not been obtained except in the one patient to be described fully elsewhere (Loizou and Smith, in preparation). In addition to the above group of patients, we identified a second group who showed white matter low attenuation on CT associated with vascular disease, but not the full syndrome of subcortical arteriosclerotic encephalopathy.

\section{Patients and methods}

All but one patient were in-patients at the National Hospital for Nervous Diseases, London. Fifteen patients were thought to have subcortical arteriosclerotic encephalopathy and all of them had been referred for evaluation of dementia and neurological deficits. Six further patients showed either clinical or CT features compatible with subcortical arteriosclerotic encephalopathy, and so were grouped separately for comparative purposes. Routine general and neurological clinical evaluation was supplemented by specific investigations including blood glucose, B12 and folate estimation, thyroid function tests, serological tests for syphilis, chest and skull radiographs, ECG, CT scan and EEG; CSF examination, psychometry and cerebral blood flow studies were carried out in the majority of patients. Cerebral blood flow was studied using the intravenous Xenon $\left({ }^{133} \mathrm{Xe}\right)$ technique. ${ }^{14}$ CT scans were taken on an EMI 1010 head unit or in the 5005 body scanner using a $160 \times 160$ matrix. Follow-up was possible in 10 patients.

\section{Results}

GROUP A: PATIENTS WITH SUBCORTICAL ARTERIOSCLEROTIC ENCEPHALOPATHY

\section{Clinical features}

The clinical features of the 15 patients are summarised in table 1 . There were nine males and six females. The age at onset of symptoms ranged from 26 to 72 years in males (in seven the range was 52 to 64 years), and 55 to 75 years in females. The duration of illness ranged between 1 to 23 years in males ( 1 to 12 years in eight of them) and 1 to 4 years in females. Hypertension requiring treatment was present in 12 patients; marginal hypertension was present in patient No 7 and two patients (Nos 1 and 13, table 1) were normotensive, with blood pressure up to $150 / 95 \mathrm{mmHg}$. The mode of presentation in six patients was with acute or resolving strokes, occurring on a background of, or associated with the development of dementia. In two patients presentation was with subacute neurological deficits (gait ataxia or apraxia) occurring on a background of dementia. In seven patients dementia was the presenting feature; in four of them it was associated with subacute progression of neurological deficits and in three it was associated with acute strokes.

The clinical recognition of these 15 patients was based on the presence of a number of the following features: (1) age at onset between 50 to 70 years, though at times earlier or later on in life, with a relatively lengthy course, (2) established hypertension or atheromatous vascular disease, (3) focal neurological deficits occurring acutely, with improvement, or developing subacutely. The latter were manifested mainly as disturbances of gait (pyramidal, extrapyramidal motor deficit, ataxia or apraxia), dysarthria and clumsiness of the arms. Unilateral or more commonly bilateral motor and occasionally sensory signs, extensor plantar responses and spastic dysarthria would be present; at times the motor deficit would amount to the full picture of pseudobulbar palsy, (4) dementia presenting with insidious memory loss and progressing to a global intellectual impairment, often less incapacitating than the motor deficit, up to the time of observation, (5) psychological disturbances ranging from loss of interest, lack of drive, mild depression to intolerable alteration in mood and personal habits, (6) stepwise deterioration in motor performance and mental functions.

\section{Laboratory investigations}

All patients had normal haematological and biochemical findings and negative tests for syphilis (except one patient with inactive, old infection). Chest radiographs showed cardiomegaly or unfolding of the aorta in five patients. Electrocardiography showed ischaemic changes, left ventricular hypertrophy or minor arrhythmias in eight patients. Cerebrospinal fluid examination was normal in eight patients, showed elevated protein 
$(0.73$ to $1.11 \mathrm{~g} / \mathrm{l})$ in three patients and was not examined in four patients. The EEG showed moderate or marked slow wave activity bilaterally in 12 patients; it showed focal abnormalities in left or right hemisphere in two patients and was not carried out in one patient.

\section{Psychometry}

Psychometric testing was carried out in all these patients. All showed a verbal and performance IQ well below the expected age specific range and premorbid level (Weschler Adult Intelligence Scale). No specific features could be ascertained, the dementia being global and affecting verbal, visual and cognitive functions. There was, however, fairly good correlation between verbal or performance defects and distribution of lesions in the white matter, or infarcts as revealed by CT scan (table 2).

\section{Cerebral blood flow}

Cerebral blood flow studies were carried out in 11 out of 15 patients. All but one showed reduction in the mean cerebral fast flow (indicating flow through grey matter) and there was also distortion of the regional flow patterns (table 2). Such regional reductions could be correlated with areas of cerebral infarction as shown on CT scan. White matter flows were also reduced in all these patients, but the technique used does not allow for accurate separate assessment of this subcortical flow. In three patients the cerebral blood flow study was repeated after a reriod of 6 to 10 months when there had been slight but definite deterioration in the dementia and motor deficits. In two patients there was no change in cerebral blood flow while in one there was further reduction in the mean and regional flows.

\section{CT scan findings}

Characteristic features seen on the CT scans of patients with subcortical arteriosclerotic encephalopathy consisted of the following (table 2, and fig 1).
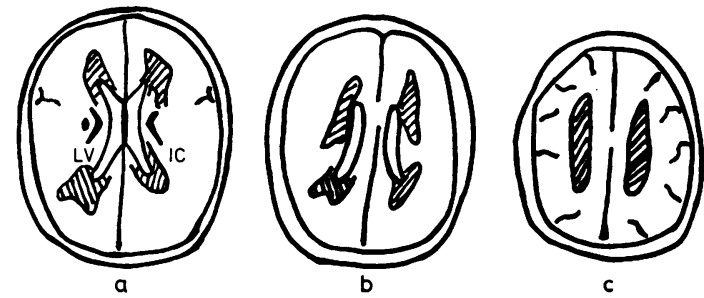

Fig 1 Diagrammatic axial CT scan sections. Cross-hatched areas indicate white matter low attenuation. In fig la there is a left putaminal infarct. $I C=$ Internal Capsule, $L V=$ Lateral Ventricle.
(1) White matter low attenuation was present bilaterally and symmetrically in all patients. The areas affected were the frontal and parietal regions in 10 patients; frontal areas only in two patients; frontal parietal and occipital in one patient; frontal, parietal and temporal in two paticnts. Thus the brunt of the disease seems to fall on the white matter of the frontal and parietal lobes in these patients (figs 2-6).

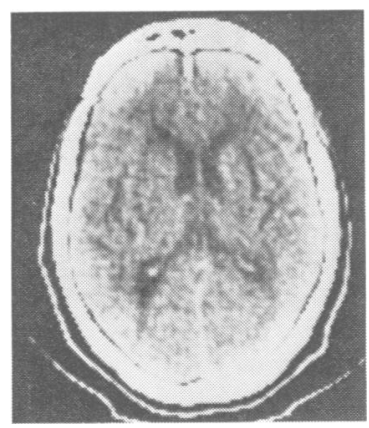

Fig 2 Unenhanced scan of patient 9 showing white matter low attenuation in the frontal regions and linear capsular infarcts.
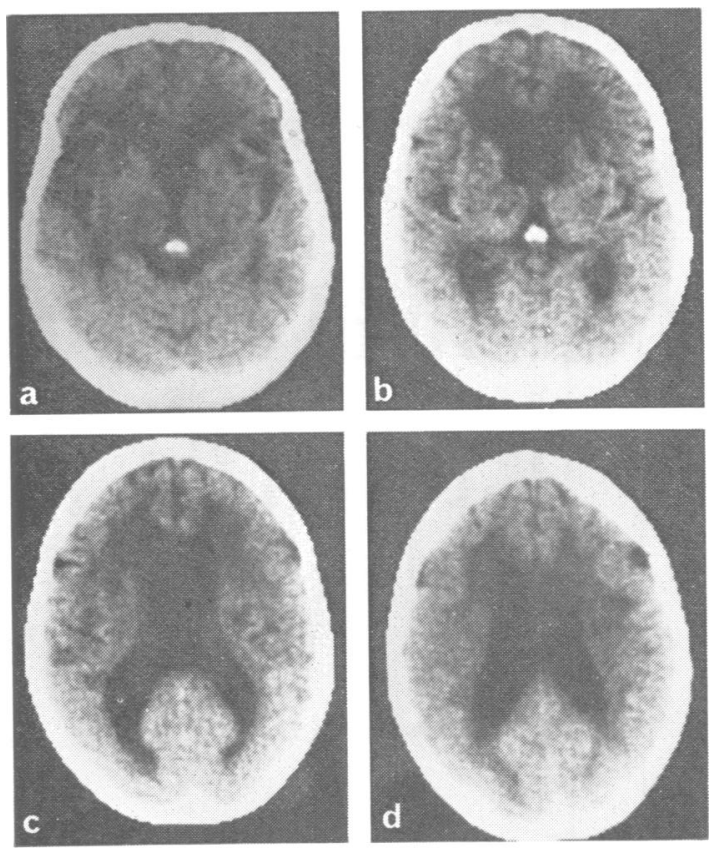

Fig 3a-d Patient 3. Unenhanced scans showing white matter low attenuation in frontal and parietal regions, lacunes in the internal capsules and moderate ventricular dilatation. 

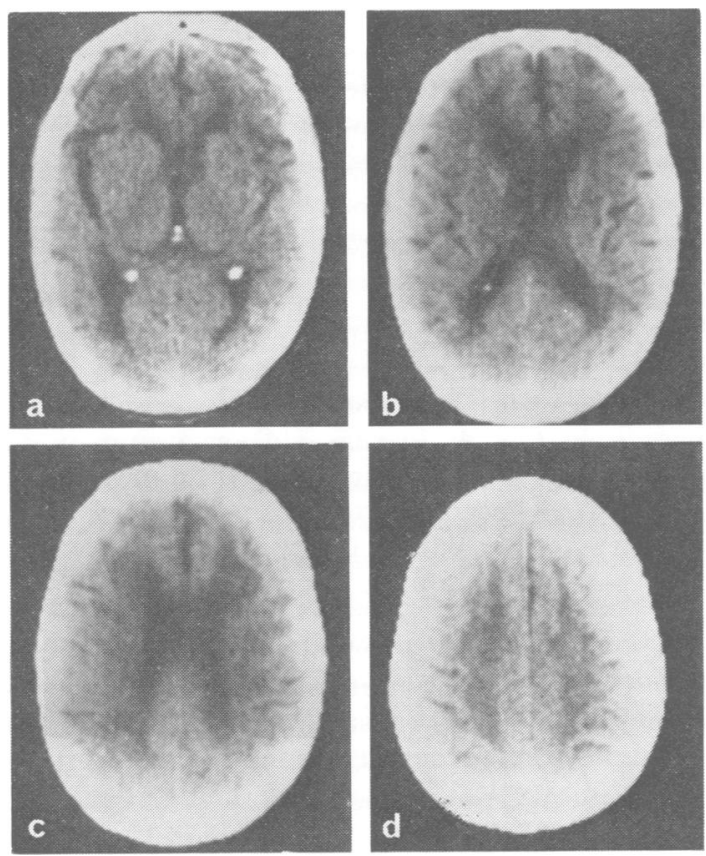

Fig 4a-d Unenhanced scans of patient 15. White matter low attenuation in the frontal parietal and centrum semiovale regions is shown. There is mild cortical atrophy and moderate ventricular dilatation.
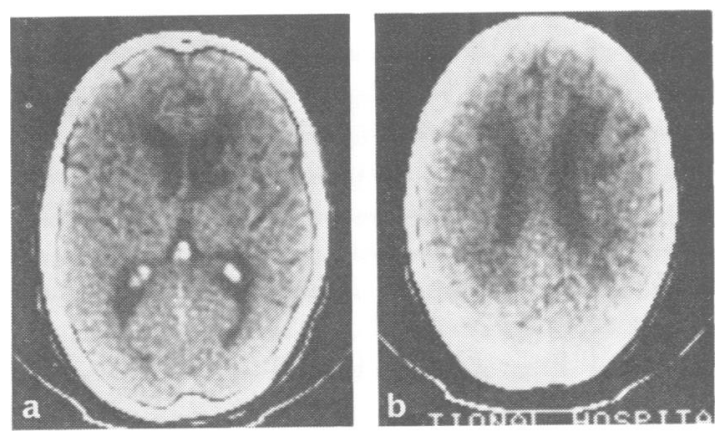

Fig 5a-b CT scans of patient 2. There is white matter low attenuation in the frontal and parietal regions, a right capsular infarct and minimal ventricular dilatation.

(2) Cortical infarcts were seen in three patients corresponding to episodes of stroke-like deficits.

(3) Subcortical lacunar infarcts were seen in nine patients, unilateral in four and bilateral in five patients, involving the internal capsules and adjacent basal ganglia.
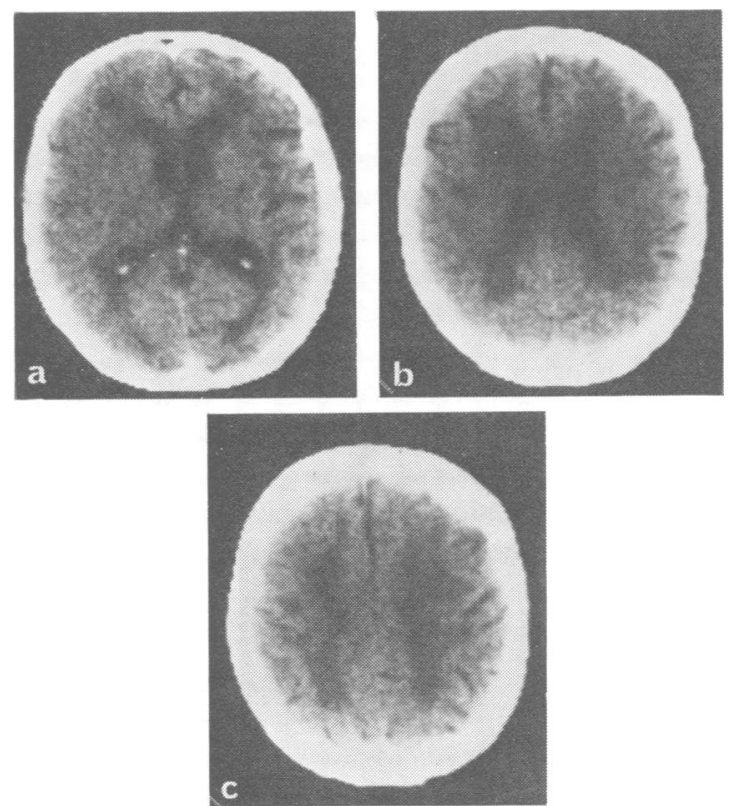

Fig 6a-c Patient 11. CT scans show white matter low attenuation in frontal, parietal and centrum semiovale regions, a right temporal lobe infarct and moderate ventricular dilatation.

(4) Dilatation of the $3 r d$ and lateral ventricles as assessed by visual inspection was seen in 12 patients and was marginal in two patients. The Evan's ratio ranged from 0.27 to 0.4 with a mean of 0.32 . On the whole ventricular dilatation was mild to moderate.

(5) Cortical sulci were normal in six patients (considering the age) and mildly to moderately dilated in nine patients.

(6) Brain swelling was not present in any case and intravenous contrast material (sodium iothalamate) did not enhance any lesions. Repeat CT scans were available in five patients. In one patient the second CT scan taken 3 years and 10 months after the first, showed the development of white matter low attenuation coinciding with deterioration in his intellect and gait ataxia. Slight to moderate progression of the white matter low attenuation was seen in three patients over a period of 6 to 18 months coincident with mental and motor deterioration, despite hypotensive medication.

\section{Neuropathological features}

Two patients died in the course of this study but only one of them had post mortem examination, 
and the findings will be described separately (Loizou and Smith, in preparation).

GROUP B: PATIENTS WITH CT AND CLINICAL

FEATURES RESEMBLING SUBCORTICAL

ARTERIOSCLEROTIC ENCEPHALOPATHY

Table 3 summarises the features of this group of six patients. Patients 1 and 2 had hypertension, dementia and neurological deficits with white matter low attenuation on CT scan, compatible with subcortical arteriosclerotic encephalopathy, but are not included in the main group because of lack of full laboratory studies. Patient 3 may exemplify the early stages of subcortical arteriosclerotic encephalopathy. He presented with two episodes of dizziness, unsteadiness and shuffling gait lasting 1-2 weeks, in 2 years; he was mildly hypertensive on no treatment, and although not demented, psychometry showed significant discrepancy between verbal and visuo-spatial executive skills. Cerebral blood flow studies showed reduced mean and regional flows (especially affecting the right temporoparietal regions) and CT showed white matter low attenuation in frontal and posterior parietal regions. Patient four presented with hypertension and a cerebellar haematoma; the CT scan showed white matter low attenuation which only partially resolved after evacuation of the haematoma. Cerebral blood flow was normal. There were no clinical features to suggest subcortical arteriosclerotic encephalopathy and up to 18 months later her only deficit was residual cerebellar ataxia; repeat CT scan at this stage was not available, the patient having moved to another area. It is possible that the white matter low attenuation in this patient was due to oedema and hypertension, rather than to white matter demyelination or gliosis. Further support for this view is provided by patient 5 , who presented with an acute stroke (dysphasia) and hypertension; the CT scan showed a left temporal infarct and white matter low attenuation in the frontal and parietal regions, the latter resolving considerably, after treatment with hypotensive medication for 2 weeks.

Patient six presented with giant cell arteritis for which she was treated with oral prednisolone. Over a period of 3 years she developed unsteadiness of gait with incoordination of the lower limbs and the CT scan showed white matter low attenuation in frontal, parietal and occipital regions, with no change over a 2 year period. Follow-up will show whether she will develop any other neurological features compatible with subcortical arteriosclerotic encephalopathy.

\section{Discussion}

In this study we attempted to identify during life a group of patients, who were likely to have the neuropathological process of subcortical arteriosclerotic encephalopathy. The diagnostic process was based on a set of clinical and CT scan criteria, which derived from the descriptions in the literature $^{51213}$ and from a case studied clinically, radiologically and pathologically by one of us (Loizou and Smith, in preparation). The main clinical features were of a slowly evolving dementia, in the 50-70 year old age group, of ten associated with changes in affect, focal or bilateral neurological deficits of acute or subacute nature and cerebrovascular aetiology, hypertensive in most patients or simply arteriosclerotic in some. The correlated CT scan features were those of generally symmetrical white matter low attenuation in frontal and parietal regions, sometimes including the external capsule region, and less often in temporal and occipital regions, with subcortical lacunes or cortical infarcts and mild to moderate cerebral atrophy. A predominantly extrapyramidal presentation ${ }^{15}$ was not encountered in our study but seizures were seen in two of our patients. ${ }^{2} 51213$

Subcortical arteriosclerotic encephalopathy should be differentiated both clinically and radiologically from similar conditions. There is a great similarity between subcortical arteriosclerotic encephalopathy and the clinical syndrome of vascular pseudobulbar palsy, the pathology of which is the lacunar state (état lacunaire). ${ }^{1-20}$ Differentiating features may be the subacute accumulation of deficits in subcortical arteriosclerotic encephalopathy associated with white matter low attenuation on CT. Cases 4 and 9 (table 1) exemplify this point. It may well be that the two conditions represent different stages in the natural history of subcortical arteriosclerosis. Multi-infarct dementia, due to accumulation of cerebral infarcts from extracranial thromboembolic disease or intracranial small vessel disease $^{32122}$ may present in an identical way. Five of our patients were initially assessed on this working diagnosis; subcortical arteriosclerotic encephalopathy was diagnosed on the basis of subacute deficits and the presence of white matter low attenuation on CT, which has not been noted in multi-infarct dementia. ${ }^{23} 24$ The correlation between accumulation of subacute deficits and dementia with white matter low attenuation was seen in almost all patients; in patient one (table 1) dysarthria and ataxic paraparesis developed slowly over 4 years and coincided with the appearance 


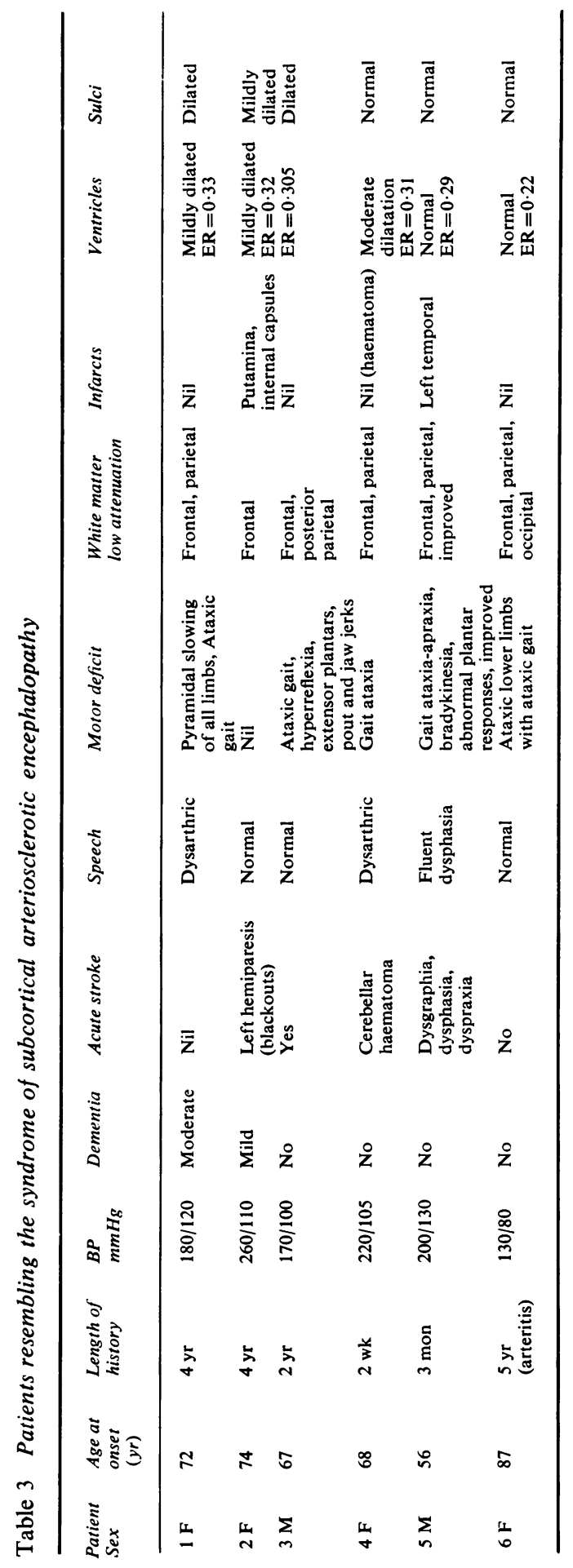


of white matter low attenuation in the frontal lobes. Other vascular conditions that may present in a similar way are watershed infarction and Moya Moya disease, but both these have characteristic clinical and radiological features.

The CT scan appearances of subcortical arteriosclerotic encephalopathy are characteristic but not absolutely diagnostic. Differentiation must be made from somewhat similar bilateral white matter low attenuation, which may be seen rarely in this age group, in association with conditions not primarily of vascular origin. ${ }^{25}$ These include advanced multiple sclerosis in which the plaques are generally asymmetrical; posttraumatic microcystic change and demyelination which is generally most pronounced in the inferior frontal regions; cerebral oedema in which the low density is ill defined and accompained by brain swelling, and rare toxic metabolic disorders, such as amyloid disease, and acute disseminated encephalomyelopathy. Such conditions may have special features of their own apart from white matter low attenuation, which will aid further differentiation. White matter low attenuation may be seen infrequently in the CT of apparently normal elderly people, though not as extensive and symmetrical as in patients with subcortical arteriosclerotic encephalopathy. ${ }^{13}$ Reduced attenuation adjacent to the frontal horns and occasionally the temporal and occipital horns is also well known in obstructive and communicating hydrocephalus, due to transependymal fluid leakage. ${ }^{26}$ Subcortical arteriosclerotic encephalopathy can be differentiated from communicating "normal pressure hydrocephalus" with which it shares the features of ventricular dilatation and mild cortical atrophy by the invariable presence of more laterally extending white matter low attenuation in frontal and parietal lobes, and the frequent occurrence of infarcts. Without CT studies the two conditions may be difficult to differentiate and lead to ineffective shunting procedures. ${ }^{1027}$

Other vascular conditions may give rise to diagnostic difficulties on CT scan. Watershed infarction due to bilateral internal or common carotid occlusion or stenosis may predominantly affect the frontal and parietal white matter at the junction of the anterior and middle cerebral supplies and extend to the periventricular regions. ${ }^{28}$ Angiography may be necessary to elucidate the diagnosis. In hypertensive encephalopathy, low density may occur in both grey and white matter on the basis of microinfarcts and petechial haemorrhages associated with cerebral oedema ${ }^{29}$; the reversible nature of this white matter low attenuation and the absence of cortical atrophy and infarcts of a size visible on CT will differentiate it from cases of subcortical arteriosclerotic encephalopathy. The CT scan appearances of état lacunaire have not been correlated in a large series with neuropathology. It may be anticipated that they would consist of mild cerebral atrophy in combination with subcortical infarcts, similar to, but more severe than in the lacunar syndrome ${ }^{30} 31$; white matter low attenuation would differentiate in favour of subcortical arteriosclerotic encephalopathy. The pathological condition of état criblé, refers to multiple small cavities in the white matter ${ }^{32} 33$ but it has no known clinical correlate, except when it occurs with état lacunaire in pseudobulbar palsy. Valentine, Kendall and Mosely ${ }^{25}$ related the CT changes of white matter low attenuation in a group of patients with hypertension and dementia to the presumed pathological state of état criblé, without histological support. It is possible that some of their patients were examples of subcortical arteriosclerotic encephalopathy, though none of them had infarcts, in addition to the other features of white matter low attenuation, dilated ventricles and mild cortical atrophy. It is evident that further neuropathological correlation in their patients and in the present series is necessary for final diagnosis. Extensive white matter low attenuation may occasionally accompany confusion or dementia in polycythaemia. ${ }^{29}$

The CT scan appearances of subcortical arteriosclerotic encephalopathy would be expected to occur uncommonly. In a recent study, ${ }^{13}$ six cases were found in $3500 \mathrm{CT}$ examinations; we encountered six cases in 1979 and eight in 1980 , out of a total of 4930 and 4560 scans (up to the time of completion of the study), respectively.

Our attempt to define characteristic psychometric features of patients with subcortical arteriosclerotic encephalopathy did not yield any specific information. There was good correlation between psychometric deficits and presence of white matter low attenuation or infarcts in specific areas as seen on CT scans. Where it was possible to repeat the psychometric tests after an interval, psychometric deterioration was shown to parallel the clinical deterioration and the progression of white matter low attenuation (patients 1, 3, 6, 11, table 1).

Cerebral blood flow studies showed abnormal low flows in all but one patient. The technique used does not allow accurate measurement of white matter flow, which would have been the major area of interest. The fact, however, that in most patients fast flow measurements, which indicate flow through the cortical grey matter, were 
reduced is a significant indication that atherosclerotic disease involves the cortical pial vessels to an extent that may impair flow in their watershed area with the white matter perforating vessels. It should be noted that these CBF findings are very similar to those seen in patients with multi-infarct dementia. ${ }^{34}{ }^{35}$.

The EEG changes seen in our patients consisted of generalised or in some cases focal slow wave abnormalities. Periodic complexes such as reported in single case reports ${ }^{1011}$ have not been encountered.

The aetiology of subcortical arteriosclerotic encephalopathy is atherosclerosis with superadded hypertensive changes affecting the intracranial cerebral arteries; medial hypertrophy and lipohyalinosis affect predominantly the perforating vessels to the white matter. These changes lead to narrowing and occlusion of the lumina especially at the sites of origin of capillaries. ${ }^{1568121336^{-38}}$ The resulting microscopic features are those of microinfarction and demyelination, focal or diffuse, affecting the frontal, parietal, temporal or occipital white matter, with associated gliosis. The periventricular regions tend to be affected most and the subcortical $U$ fibres tend to be spared, as is the cortex. The ventricles show compensatory dilatation.

The pathogenesis of white matter degeneration has been related to two basic changes. The first is the change in the wall of the perforating vessels ${ }^{39}$; the second is either chronic hypoperfusion and ischaemia $^{37}$ or focal cerebral oedema with hypoxia. ${ }^{4041}$ Our blood flow studies indicate involvement of the cortical vessels in the arteriosclerotic process and indeed a number of authors have reported this neuropathologically. ${ }^{42}$ We suggest, therefore, that the white matter changes are due to chronic ischaemia in the watershed area between the cortical medullary arteries and the long perforating arteries to the white matter. Wodar $\mathrm{z}^{28}$ has suggested such a mechanism in watershed infarction. Hypertension probably accentuates the basic arteriosclerotic process. It is of interest that in a recent review of 20 patients with malignant hypertension and 50 on chronic dialysis programmes, none was encountered who showed white matter low attenuation on CT scan $^{13}$; this implies that hypertension may not be the only factor in the pathogenesis of white matter low attenuation, which is the CT correlate of white matter degeneration.

In conclusion we have defined the clinical features and CT appearances of patients with the diagnosis of subcortical arteriosclerotic encephalo- pathy. Both the clinical and CT features must be differentiated from similar conditions, but when taken together they are virtually diagnostic of subcortical arteriosclerotic encephalopathy.

We gratefully acknowledge the cooperation of our clinical colleagues at the National Hospitals for Nervous Diseases and of Dr Elizabeth Warrington for the psychometric assessments. Dr M Brown performed some of the cerebral blood flow measurements with the technical assistance of Miss S Redmond. Miss B Laatz and Miss J Cox gave invaluable secretarial help.

\section{References}

1 Olszewski J. Subcortical arteriosclerotic encephalopathy. World Neurol 1962; 3:359-75.

2 Binswanger $\mathrm{O}$. Die Abgrenzung der allgemeinen progressiven paralyse. Berl Klin Wochenschr $1894 ; 31: 1103-5,1137-9,1180-6$.

3 Hachinski VC, Lassen NA, Marshall J. Multiinfarct dementia. A cause of mental deterioration in the elderly. Lancet $1974 ; 2: 207-10$.

4 Biemond A. On Binswanger's subcortical arteriosclerotic encephalopathy and the possibility of its clinical recognition. Psychiatria Neurolog Neurochirurg 1970; 73:413-7.

5 Caplan LR, Schoene WC. Clinical features of subcortical arteriosclerotic encephalopathy (Binswanger disease). Neurology (Minneap), 1978; 28: 1206-15.

6 Farnell FJ, Globus JH. Chronic progressive vascular subcortical encephalopathy. Arch Neurol Psychiatry 1932; 27:593-604.

7 Biggart JH. Pathology of the nervous system. Edinburgh: Livingstone, 1936, 56-7.

8 Davison C. Progressive subcortical encephalopathy. J Neuropathol Exp Neurol 1942; 1:42-8.

9 Aronson SM, Perl DP. Clinical neuropathological conference. Dis Nerv Syst 1974; 35:286-91.

10 Burger PC, Burch JG, Kunze U. Subcortical arteriosclerotic encephalopathy (Binswanger's disease) Stroke 1976; 7:626-31.

11 White JC. Periodic EEG activity in subcortical arteriosclerotic encephalopathy (Binswanger's type). Arch Neurol (Chicago) 1979; 36:485-9.

12 Rosenberg GA, Kornfeld M, Stovring J, Bicknell JM. Subcortical arteriosclerotic encephalopathy (Binswanger): Computerised Tomography. Neurology (Minneap) 1979; 29:1102-6.

13 Zeumer H, Schonsky B, Sturm KW. Predominant white matter involvement in subcortical arteriosclerotic encephalopathy (Binswanger Disease). J Comput Assist Tomograph 1980; 4(1):14-9.

14 Thomas DJ, Zilkha E, Redmond S et al. An intravenous ${ }^{133}$ Xenon clearance technique for measuring cerebral blood flow. J Neurol Sci 1979; 40:53-63. 
15 Ishino $H$, Higashi $H$, Hayahara $T$, Ikeda $H$, Otsuki S. A case of subcortical arteriosclerotic encephalopathy (Binswanger's disease). Folia Psychiatr Neurol Jpn 1972; 26:39-44.

16 Marie P. Des foyers lacunaires de desintegration et de differents autres états cavitaires du cerveau. Rev Med (Paris) 1901; 21:281.

17 Hughes W, Dodgson MCH, McLennan DC. Chronic cerebral hypertensive disease. Lancet 1954; 2:770-4.

18 Fisher CM. Lacunes: small deep cerebral infarcts. Neurology (Minneap) 1965; 15:774-84.

19 Fisher CM. The arterial lesions underlying lacunes. Acta Neuropathol (Berlin) 1969; 12:1-15.

20 Gautier JC. Cerebral ischaemia in hypertension. In: Cerebral Arterial Disease. Ross Russell RW ed. Edinburgh: Churchill Livingstone, 1976, 181209.

21 Tomlinson BE, Blessed G, Roth M. Observations on the brains of demented old people. J Neurol Sci 1970; 11:205-42.

22 Tomlinson BE. The pathology of dementia. In: Wells Ch E ed. Dementia Contemporary Neurology Series. Ed 2, Philadelphia: FA Davis Co, 1977; 113-53.

23 Radu EW, du Boulay GH, Harrison MJG, Thomas DJ. Comparison of angiographic and CT findings between patients with multi-infarct dementia and those with dementia due to primary neuronal degeneration. Neuroradiology 1978; 16: 113-5.

24 Ladurner G, Sager WD, Iliff LD, Lechner H. A correlation of clinical findings and CT in ischaemic cerebrovascular disease. Eur Neurol 1979; 18: 281-8.

25 Valentine AR, Moseley IF, Kendall BE. White matter abnormality in cerebral atrophy: clinicoradiological correlations. J Neurol Neurosurg Psychiatry 1980; 43:139-42.

26 Moseley IF, Radu EW. Factors influencing the development of periventricular lucencies in patients with raised intracranial pressure. Neuroradiology 1979; 17:65-9.

27 Earnest MP, Fahn S, Karp JH, Rowland LP. Normal pressure hydrocephalus and hypertensive cerebrovascular disease. Arch Neurol (Chicago) 1974; 31:262-6.

28 Wodarz R. Watershed infarctions and computed tomography. A topographical study in cases with stenosis or occlusion of the carotid artery. Neuroradiology 1980; 19:245-8.

29 Kendall BE, Claveria LE, Quiroga W. In: du Boulay GH, Moseley IF eds. CAT in leukodys- trophy and neuronal degeneration. European seminar on computerised axial tomography in clinical practice. Berlin: Springer-Verlag, 1977, 191-202.

30 Weisberg LA. Computed tomography and pure motor hemiparesis. Neurology (Minneap) 1979; 29:490-5.

31 Nelson RF, Pullicino P, Kendall BE, Marshall J. Computed tomography in patients presenting with lacunar syndromes. Stroke 1980; 11:256-61.

32 Hughes W. Origin of lacunes. Lancet 1965; 2 : 19-21.

33 Cole FM, Yates PO. Comparative incidence of cerebrovascular lesions in normotensive and hypertensive patients. Neurology (Minneap) 1968; 18:255-9.

34 Hachinski VC, Iliff LD, Zilkha E, du Boulay GH, McAllister VL, Marshall J, Ross Russell RW, Symon L. Cerebral blood flow in dementia. Arch Neurol (Chicago) 1975; 32:632-7.

35 Harrison MJG, Thomas DJ, du Boulay GH, Marshall J. Multi-infarct dementia. J Neurol Sci 1979; 40:97-103.

36 Bruetsch WL. The myth of Binswanger disease (Discussion). J Neuropathol Exp Neurol 1961; 29:313.

37 Jellinger K, Neumayer E. Progressive subcorticale vasculare encephalopathie Binswanger. Eine Klinisch-Neuropathologische Studie. Arch Psychiatr Nervenkr 1964; 205:523-54.

38 Iglesias-Rozas JR, Ebhardt G. Alterations of microvasculature in progressive subcortical encephalopathy (Binswanger). In: Cervos-Navarro J ed, Pathology of cerebral microcirculation. Berlin: Walter de Gruyter, 1974; 454-61.

39 Okeda R. Correlative morphometric studies of cerebral arteries in Binswanger's encephalopathy and hypertensive encephalopathy. Acta Neuropathol (Berl) 1973; 26:23-43.

40 Feigin I, Popoff N. Neuropathological changes late in cerebral oedema: the relationship to trauma, hypertensive disease and Binswanger's encephalopathy. J Neuropathol Exp Neurol 1963; 22:500-11.

41 Feigin I, Budzilovich G, Weinberg S, Ogata J. Degeneration of white matter in hypoxia, acidosis and oedema. J Neuropathol Exp Neurol 1973; 32:125-43.

42 Crompton RM. Pathology of degenerative cerebral arterial disease. In: Ross Russell RW ed, Cerebral Arterial Disease, Edinburgh: Churchill Livingstone, 1976; 40-56. 